\title{
A Model Based Approach for Multi-lead ECG Array Layout Selection
}

\author{
Christoph Hintermüller, Michael Seger, Bernhard Pfeifer, \\ Gerald Fischer, and Bernhard Tilg \\ Institute for Biomedical Engineering, \\ University for Health Sciences, Medical Informatics and Technology, \\ Eduard Wallnöfer Zentrum I, 6060 Hall in Tyrol, Austria \\ Telephone: 0043/50/8648/3820, Fax: 0043/50/8648/3850
}

\begin{abstract}
In this study an approach for testing electrode array schemes with respect to their ability to improve the resolution of methods for activation time imaging is proposed. First local linear dependency maps are computed using a virtual array method. These maps depict the torso surface areas where the body surface potential is most sensitive to changes in the transmembrane potential. The optimal number and position of the electrodes within the sensitive body surface regions was selected by constructing effort gain (EG) plots. Such a plot depicts the relative attainable rank of the leadfield matrix in relation to the increase in number of electrodes required to build the electrode array.

From the sensitivity maps it was found that the BSP is most sensitive to changes in TMP on the upper left frontal and dorsal body surface. The EG analysis revealed that the optimal array meeting clinical requirements and improving the resolution of activation time imaging consists of 125 electrodes.
\end{abstract}

\section{Introduction}

Here methods for selecting an electrode array scheme maximizing the information content in body surface potential recordings are proposed.

Observations upon linear dependency between electrodes are used to locate areas of high information content in the BSP patterns by calculating so called local linear dependency (LLD) maps. The volume conductor model required for the LLD analysis was extracted from patient individual magnetic resonance imaging (MRI) scans, recorded prior to the clinical treatment the patient had to undergo. As depicted in Fig. 1 it basically consists of four different compartments, the chest surface, the left and right lungs, the ventricular and atrial myocardium and the associated bloodmasses. Further, it contains information upon the electric conductivity of the different compartments and the positions of the electrodes the BSP is recorded with. Especially locating the posterior electrodes is a rather tedious procedure as their position is reconstructed from the MRI scans by locating and identifying vitamin E marker capsules. Whereas the positions of the frontal ones can be recorded using a digital position tracking 
system such as FASTRACK (Polhemus Inc, Colchester, USA). Recording the posterior electrodes with the FASTRACK system would require the patient to sit upright which usually is not possible. Further, the proper electrical contact of all electrodes to the body surface of the patient has to be considered critical. In case of improper contact the signal to noise ratio (SNR) is significantly lower than the required level (in our case $>40 \mathrm{~dB}$ ).

The BSP recordings were used to estimate the electric activation for the heart by computing the activation time as described in [1, 2, 3, 4, 5]. The information upon the electric sources of the heart was noninvasively obtained by Recording the BSP. The BSP is used in combination with an individual volume conductor model to solve the underlying inverse problem. Before solving this inverse problem, the type of information which is determined in the inverse problem has to be specified. For detecting the earliest activation of the heart an AT imaging method will be the best choice. In any case the changes in the TMP related to the activation of the heart cause changes in the BSP. The corresponding solutions are sensitive to the recording location of the BSP.This sensitivity is caused by varying linear dependencies in the equation system obtained for the forward electrocardiographic problem. In case of high linear dependency the BSP contains less additional information while BSP recorded from locations with low linear dependency contains more independent information about cardiac electric activity.

\section{Methods}

For solving the electrocardiographic forward and inverse problem a patient individual volume conductor model is used. Such a model the patients torso, lungs, ventricles, atria and associated blood masses and the location of the electrodes. The different compartments have been segmented using methods and tools described in [6]. The volume conductor includes the compartment conductivities $\kappa$ and $\kappa_{\mathrm{H}}$ for the heart. Using discrete boundaries surfaces with the interpolation coefficients $n_{i}, n_{j, \mathrm{H}}$ and the potentials $V_{i}(t), \varphi_{\mathrm{m}, j}(t)$ and applying the Wilson Terminal formulation as done by [7] the relation between the TMP $\varphi_{\mathrm{m}}$ on the heart surface and the BSP $V$ measured by the electrodes can be expressed as

$$
V_{\mathrm{e}}(t)=\mathbf{I N} \varphi_{\mathrm{m}}(t)=\mathbf{L} \varphi_{\mathrm{m}}(t) .
$$

The leadfield matrix $\mathbf{L}$ in (1) consists of the interpolation matrix $\mathbf{I}$ interpolating the discrete BSP to gain the electrode potential and the node field matrix $\mathbf{N}$ describing the patients volume conductor.

\subsection{Local Linear Dependency}

In order to identify the sensitive areas a virtual array (VA) method which is similar to the virtual displacement method was applied. The distance $\Delta d$ between the virtual electrodes and the central one located at the point of investigation $P_{\mathrm{ci}}$ is not infinitesimally small, which would be the case for the virtual displacement 


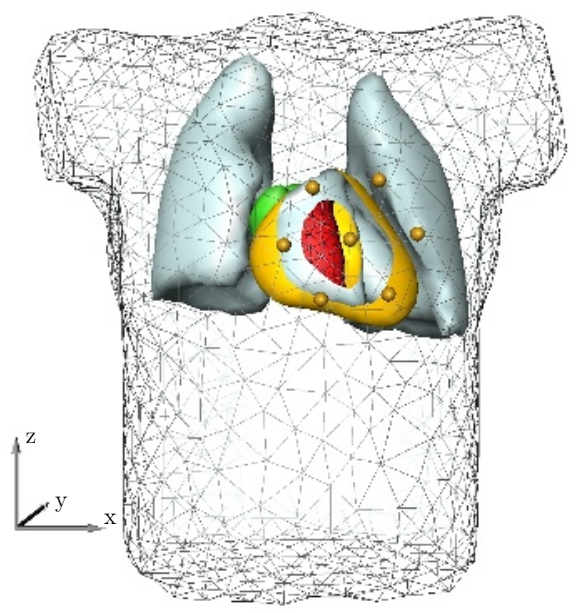

Fig. 1. Volume conductor model consisting of the atria, the ventricles, the corresponding blood masses, the lungs and the chest. A seven lead virtual array, centered at a node of the body surface mesh, is depicted. The right ventricle is cut open to show the corresponding blood mass.

method. Figure 1 shows such a VA with $P_{\text {ci }}$ equal to a node of the triangular body surface mesh.

From the leadfield matrix $\mathbf{L}_{\mathrm{virt}}$ of a VA a LLD measure $l$ with

$$
l=\frac{s_{\max }}{s_{\min }}
$$

was computed. In (2) $s_{\min }$ and $s_{\max }$ are the smallest and largest singular value of $\mathbf{L}_{\mathrm{virt}}$. The singular values $s_{i}$ are obtained by singular value decomposition (SVD) of $\mathbf{L}$ as follows:

$$
\mathbf{L}=\mathbf{U} \mathbf{\Sigma} \mathbf{Q}^{\mathrm{T}} .
$$

The rectangular matrix $\mathbf{U}$ contains the eigenvectors spanning the orthonormal basis of the electrode (row) space. The diagonal matrix $\boldsymbol{\Sigma}$ contains the singular values and $\mathbf{Q}$ contains the eigenvectors spanning the orthonormal basis of the source (column) space.

\subsection{Attainable Rank}

In addition to the VA based LLD maps the singular values $s_{i}$ for the leadfield matrix $\mathbf{L}$ of an electrode array were computed. According to (11) and (3) the TMP $\varphi_{\mathrm{m}}(t)$ related to source space $\mathbf{Q}$ is mapped by $\mathbf{L}$ onto the BSP in electrode space $\mathbf{U}$. Thus, $\varphi_{\mathrm{m}}(t)$ can be expressed as a linear combination of the source eigenvectors $q_{i}$ spanning $\mathbf{Q}$ as

$$
\varphi_{\mathrm{m}}(t)=\Delta \varphi_{\mathrm{m}} \sum_{i} \alpha_{i}(t) q_{i}
$$


with the action potential amplitude $\Delta \varphi_{\mathrm{m}}$. The time dependent coefficients $\alpha_{i}(t)$ map $q_{i}$ onto $\varphi_{\mathrm{m}}(t)$. They range from $\alpha_{i}(t)=0$ corresponding to a total suppression of the spatial frequencies contained in $q_{i}$ and $\alpha_{i}(t)=1$ fully mapping $q_{i}$ onto $\varphi_{\mathrm{m}}(t)$.

For estimating the attainable rank $R$ of $\mathbf{L}$ a "detector" or "receiver criterion" based upon the following assumptions was applied:

1. The source distribution $\varphi_{\mathrm{m}}(t)$ is equal to $\varphi_{\mathrm{m}}(t)=\varphi_{\mathrm{m}}=$ const for all time instants $t$ reducing (4) to

$$
\varphi_{\mathrm{m}}=\Delta \varphi_{m} \sum_{i} \alpha_{i} q_{i}
$$

2. The source space $\mathbf{Q}$ is successively mapped onto $\varphi_{\mathrm{m}}$ by setting all $\alpha_{i}=0$ in (44) except one $\alpha_{i}=\alpha_{j}=1$. The index $j$ is used to select one specific $q_{i=j}$. It ranges from $j=1$ to the number of singular values $s_{i}$ of $\mathbf{L}$.

Applying this assumptions to (11) the norm $\left\|V_{\mathrm{e}}(j)\right\|$ of the corresponding BSP vectors $V_{\mathrm{e}}(j)$ results in

$$
\left\|V_{e}(j)\right\|=\left\|\mathbf{L} \Delta \varphi_{\mathrm{m}} q_{i=j}\right\|=s_{i=j} \Delta \varphi_{m} .
$$

A detector in general is characterized by its measurement range, the number of leads $Z$ the norm $\|\sigma\|=\sqrt{Z} \sigma_{l}$ of the noise vector $\sigma$ containing the noise levels $\sigma_{l}$ for all leads. The detector criterion uses $\left\|V_{\mathrm{e}}(j)\right\|$ and $\|\sigma\|$ to classify all $V_{\mathrm{e}}(j)$ with respect to their information content. It separates vectors $V_{\mathrm{e}}(j)$ resulting from the mapping of $\varphi_{\mathrm{m}}(j)$ to the target space $\mathbf{U}$ of $\mathbf{L}$ by (11) from vectors $V_{\mathrm{e}}(j)=\sigma$. The attainable rank $R$ of $\mathbf{L}$ can be found by comparing all $\left\|V_{\mathrm{e}}(j)\right\|$ gained by (6) with $\|\sigma\|$ applying the following condition

$$
s_{i=j+1} \Delta \varphi_{\mathrm{m}} \leq\|\sigma\|<s_{i=j} \Delta \varphi_{\mathrm{m}} .
$$

The index $j$ for which (17) holds is equal to the attainable rank $R$ of $\mathbf{L}$.

\subsection{Effort, Gain Analysis}

One possibility to compare two electrode array layouts $S_{0}$ and $S_{i}$ is to compare their attainable ranks $R_{0}, R_{i}$ and the number of electrodes $Z_{0}, Z_{i}$ used to build them. This was done by computing measures for the gain in rank $r_{i}$ and the effort $z_{i}$ when converting $S_{0}$ into $S_{i}$ by changing position and number of electrodes. The measures $z_{i}$ and $r_{i}$ are computed in terms of $Z$ and $R$ as follows:

$$
r_{i}=\frac{R_{i}}{R_{0}}, \quad z_{i}=\frac{Z_{i}}{Z_{0}} .
$$

When scheme $S_{0}$ is compared to several $S_{i}$ than the corresponding measures $r_{i}$ and $z_{i}$ can be used to construct a so called effort-gain (EG) plot. Such a plot depicts the relative increase in $R$ with respect to the relative changes in $Z$. In general the EG-plot can be used to compare the arrays $S_{i}$ to each other and select the optimal layout. 


\section{Results}

\subsection{Sensitive Body Surface Regions}

Volume conductor models for eleven patients (9 male and 2 female) having different body shape, age (between 19 and 57 years) and size were generated. Each model was segmented from MRI scans recorded prior to the electrophysiological intervention. Based on these models the patient specific LLD maps for the atria, the ventricles and the whole heart were computed. Figure 2 depicts the anterior part of these LLD maps for an individual patient. By comparing the LLD maps for all 11 patients it was found that the sensitive areas expressing low $l$ are located left frontal and dorsal between the shoulder blades. In average, the sensitive regions (gray area, Figure 2) were somehow $L$-shaped and showed a frontal size of $\sim 20 \times 30 \mathrm{~cm}$ to $\sim 25 \times 35 \mathrm{~cm}$ and $\sim 15 \times 20 \mathrm{~cm}$ to $\sim 20 \times 25 \mathrm{~cm}$ on the back.

In a first step the electrodes were arranged in two regular spaced $L$-shaped patches. In order to ensure that the sensitive regions for the atria, ventricles and the whole heart are covered best, a horizontal and vertical spacing of the electrodes equal to $4 \mathrm{~cm}$ (Fig. 2) was selected. The aim was to compare the array prototype of scheme $\mathrm{S}_{\mathrm{B}}$ to an array scheme $\mathrm{S}_{\mathrm{A}}$, initially developed at the University of Amsterdam [8]. The number of electrodes for $\mathrm{S}_{\mathrm{B}}$ was selected to be the same as for $\mathrm{S}_{\mathrm{A}}$. Due to the difficulties to extract the posterior electrode positions, as mentioned in section 1 the number of posterior electrodes had to be reduced form 21 to 11 for the layout of scheme $\mathrm{S}_{\mathrm{A}}$. Thus, $\mathrm{S}_{\mathrm{B}}$ was compared to the modified array scheme $\mathrm{S}_{\mathrm{A}}$ in the following addressed as $\mathrm{S}_{\mathrm{A}, 62}$ instead of the original scheme $\mathrm{S}_{\mathrm{A}}$ proposed by $[8$.

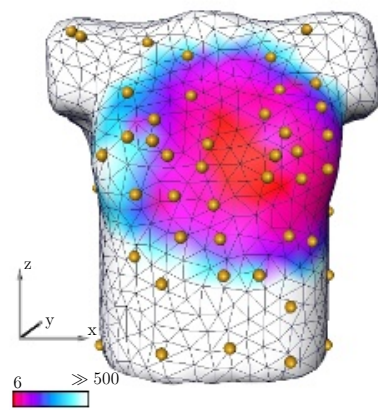

(a)

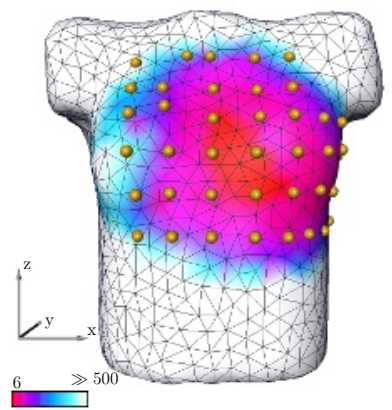

(b)

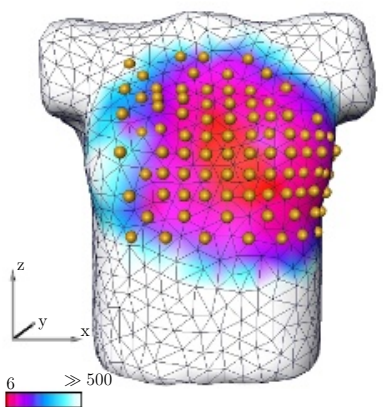

(c)

Fig. 2. The anterior linear dependency (LLD) map for an individual patient and its coverage by three different electrode arrays (spheres) (a) (b) (c). The gray surface indicates the frontal sensitive area (a) shows an idealized version $\mathrm{S}_{\mathrm{A}, 62}$ of scheme $\mathrm{S}_{\mathrm{A}}$. As it can be observed it covers the anterior region poorly. The other two arrays $\mathrm{S}_{\mathrm{B}, 62}$ (b) and $\mathrm{S}_{\mathrm{B}, 125 \mathrm{~S}}$ (c) expose a better coverage. Both are based upon the horizontal and vertical regular spaced layout introduced by scheme $\mathrm{S}_{\mathrm{B}}$. 
In detail the initial prototype $\mathrm{S}_{\mathrm{B}, 62}$ of $\mathrm{S}_{\mathrm{B}}$ consisted of 62 Electrodes (41 anterior and 21 posterior). To perform the comparison of $\mathrm{S}_{\mathrm{B}}$ with $\mathrm{S}_{\mathrm{A}}$ the leadfield matrices $\mathbf{L}_{\mathrm{S}_{\mathrm{B}}}$ and $\mathbf{L}_{\mathrm{S}_{\mathrm{A}}}$ for the atria, the ventricles and the whole heart were computed. The corresponding ranks $R$ were estimated using the detector criterion specified by (7). In addition, $\mathrm{S}_{\mathrm{B}}$ and $\mathrm{S}_{\mathrm{A}}$ were compared to a variant $\mathrm{S}_{\mathrm{A}, 59}$ of scheme $\mathrm{S}_{\mathrm{A}}$ which was recorded during the clinical procedure [5] the entire patient had to undergo.

Further, the figures for effort $m$ and gain $r$ were calculated. iFor all 11 patients the arrays $\mathrm{S}_{\mathrm{B}, 62}$ and $\mathrm{S}_{\mathrm{B}, 125 \mathrm{~S}}$ exposed larger values for $r$ than $\mathrm{S}_{\mathrm{A}, 62}$ (eg: $r=1$ for $\mathrm{S}_{\mathrm{A}, 62}, r=1.2$ for $\mathrm{S}_{\mathrm{B}, 62}$ and $r=1.76$ for $\mathrm{S}_{\mathrm{B}, 125 \mathrm{~S}}$ ). The arrays differ in their size, the body surface area they cover and the horizontal and vertical spacing of the electrodes. The scheme $\mathrm{S}_{\mathrm{A}, 62}$ covers the whole body whereas $\mathrm{S}_{\mathrm{B}, 62}$ only covers the sensitive areas. As it is suggested that a compact array is more applicable than a large one it may be concluded that $\mathrm{S}_{\mathrm{B}, 62}$ is more suitable for our purpose than scheme $\mathrm{S}_{\mathrm{A}, 62}$.

In case of the atria the values for the gain are $r=1.2$ for $\mathrm{S}_{\mathrm{B}, 62}$ and $\mathrm{S}_{\mathrm{B}, 125 \mathrm{~S}}$ and $r=1$ for $\mathrm{S}_{\mathrm{A}, 62}$ and $\mathrm{S}_{\mathrm{A}, 59}$. The numbers obtained for the whole heart are similar to the ones for the ventricles, being $r=1.07$ for $\mathrm{S}_{\mathrm{B}, 62}, r=1.64$ for $\mathrm{S}_{\mathrm{B}, 125 \mathrm{~S}}$ and $r=1$ for $\mathrm{S}_{\mathrm{A}, 62}$ and $\mathrm{S}_{\mathrm{A}, 59}$.

\subsection{Selection of Optimal Electrode Positions}

Based on these findings and the suggestions given in [9] upon the clinical applicability of the $L$-shaped scheme $\mathrm{S}_{\mathrm{B}}$ the search for the ideal number and density of electrodes was limited to $L$-shaped arrays derived from scheme $\mathrm{S}_{\mathrm{B}}$. As a consequence two groups of electrode arrays based on scheme $\mathrm{S}_{\mathrm{B}}$ generated. The

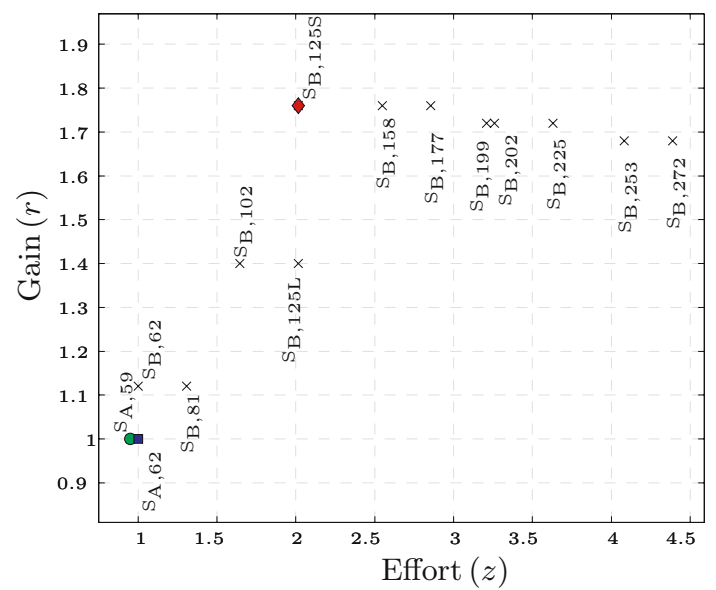

Fig. 3. Effort-gain-plot for one patient. The circular marker indicates the values for scheme $\mathrm{S}_{\mathrm{A}, 59}$ recorded in the catheter lab. Its idealized version $\mathrm{S}_{\mathrm{A}, 62}$ is depicted by the

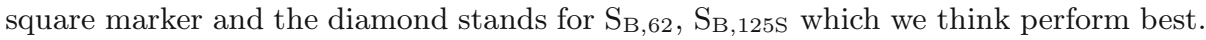
In case of an atrial source $\mathrm{S}_{\mathrm{B}, 62}$ would be sufficient whereas in case of the ventricles $\mathrm{S}_{\mathrm{B}, 125 \mathrm{~S}}$ should be the preferred choice. 
first exposed the same shape and size as $\mathrm{S}_{\mathrm{B}, 62}$ and the second was gained by expanding the size of the two $L$ shaped parts. This enlargement was achieved by adding an additional row and column at a horizontal and vertical spacing of $4 \mathrm{~cm}$ to $\mathrm{S}_{\mathrm{B}, 62}$ resulting in the 81 lead array $\mathrm{S}_{\mathrm{B}, 81}$. For both sets further arrays were gained by subdividing their horizontal and vertical spacing.

For each of the 11 patients the models for the 14 arrays were generated and the corresponding rank $R$, gain $r$, effort $m$ and the norm $\|\sigma\|$ of the detector noise vector $\sigma$ were computed. The measures for $m$ and $r$ were used to generate patient individual EG plots, Fig. 3. Further the optimal array scheme was estimated. Figure 3 depicts the EG plots for the ventricles. When repeating the EG analysis for the atria, the ventricles and the whole heart, it was found that the 125 lead array scheme labeled $\mathrm{S}_{\mathrm{B}, 125 \mathrm{~S}}$ meet most of our requirements.For the atria the 62 lead array labeled $\mathrm{S}_{\mathrm{B}, 62}$ would be sufficient.

\section{Discussion}

The results gained by the VA based LLD analysis lead to the assumption that it is possible to design an electrode array meeting the requirements given in [9. This was mainly imposed by the fact that for all patients most information about the electrical excitation of the heart can be recorded from body surface regions located near to the heart. These regions, as depicted in Fig. 2, are located central and left on the frontal and dorsal body surface. The shape of the sensitive body surface regions can be roughly approximated by an $L$-shape. Thus, it was decided to use two $L$-shaped pieces to construct an electrode array meeting the clinical requirements. For the application of the frontal part it is suggest to use the six standard ECG leads $V_{1}$ to $V_{6}$ as landmarks. And the posterior array may be aligned along the spinal cord touching the tip of the left shoulder blade with its horizontal part.

By overlaying the LLD maps with models of the electrode scheme $\mathrm{S}_{\mathrm{A}}$ as done in Fig. 2(a), it can be found that $\mathrm{S}_{\mathrm{A}, 62}$ and $\mathrm{S}_{\mathrm{A}, 59}$ do not cover the sensitive areas very well. Both expose an irregular electrode spacing and cover the patients body completely. This causes the poor coverage of the sensitive areas (colors) as can be seen in Fig. 2(a). Especially the low number of posterior electrodes, is enforced by the need to reconstruct their positions from MRI scans.

As mentioned in sec. 1 it was necessary to modify the scheme $\mathrm{S}_{\mathrm{A}, 62}$ to be able to properly locate and identify the posterior electrodes. A similar identification and location process will be necessary for the posterior electrodes of $\mathrm{S}_{\mathrm{B}, 62}$ and $\mathrm{S}_{\mathrm{B}, 125 \mathrm{~S}}$ too. Although they contain a lot more dorsal electrodes than $\mathrm{S}_{\mathrm{A}, 62}$, it may be assumed that the regular horizontal and vertical spacing supports the following approach. The MRI scans of the patient are used to locate 2 - 7 characteristic electrodes. These electrodes may mark the edges of electrode array shape or some anatomic landmarks. The position of the remaining electrodes may then be reconstructed by matching the model of the posterior array with these landmarks. Even further, this two step approach could be used to locate the frontal electrodes too, instead of the FASTRACK (Polhemus Colchester Inc, USA) locator system. 


\section{References}

1. Greensite, F.: Remote reconstruction of confined wavefront propagation. Inverse Problems 11 (1995) 361-370

2. Greensite, F.: Cardiac electromagnetic imaging as an inverse problem. Electromagnetics 21 (2001) 559-557

3. Huiskamp, G., Greensite, F.: A new method for myocardial activation imaging. IEEE Transactions on Biomedical Engineering 44 (1997) 433-446

4. Cuppen, J., Van Oosterom, A.: Model studies with inversely calculated isochrones of ventricular depolarization. IEEE Transactions on Biomedical Engineering 31 (1984) 652-659

5. Modre, R., Tilg, B., Fischer, G., Wach, P.: Noninvasive myocardial activation time imaging: A novel inverse algorithm applied to clinical ecg mapping data. IEEE Transactions on Biomedical Engineering 49 (2002) 1153-1161

6. Pfeifer, B., Seger, M., Hintermüller, C., Modre, R., Hanser, F., Tilg, B.: Patientspecific volume conductor modeling for noninvasive imaging of cardiac electrophysiology. IEEE Transactions on Medical Imaging (2005) (submitted).

7. Fischer, G., Tilg, B., Modre, R., Hanser, F., Messnarz, B., Wach, P.: On modeling the wilson terminal in the boundary and finite element method. IEEE Transactions on Biomedical Engineering 49 (2000) 217-224

8. SippensGroenewegen, A., Peeters, H.A.P., Jessurun, E.R., Linnenbank, A.C., de Medina, E.O.R., Lesh, M.D., van Hemel, N.M.: Body surface mapping during pacing at multiple sites in the human atrium. Circulation 1997 (1998) 369-380

9. Hintermüller, C., Fischer, G., Seger, M., Pfeifer, B., Hanser, F., Modre, R., Tilg, B.: Multi-lead ecg electrode array for clinical application of electrocardiographic inverse problem. In: Proceedings of the 26th Annual International Conference of the IEEE EMBS. (2004) 1941-1944 